\title{
Research on Applications of Semiconductor Laser in Medical Fields
}

\author{
Chen Jialiang \\ Taiyuan University of Technology, Taiyuan, Shanxi, China \\ jialiang21@163.com
}

Keywords: semiconductor laser; medical fields

\begin{abstract}
Due to the advantages of semiconductor laser such as small body, light weight, long life span, high efficiency, it has been used widely in the medical fields. This paper firstly introduces the function mechanism of semiconductor laser, and then analyzes the special applications in ophthalmology department, surgery department, cosmetology department and stomatology department. Finally, the broad prospect of applications of semiconductor laser in medical fields is given to provide references to relevant researchers.
\end{abstract}

\section{Function Mechanism of Semiconductor Laser}

There are two main mechanisms of semiconductor lasers in medical applications:The first is bio-stimulation mechanism. Stimulus is a concept on the biological functions. According to the biological function, autonomic nerve reflex, Pavlov's molecular biology and the principle of theory, can think that the weak interaction between laser and organism, laser is a source of stimulation, and the organism has special feel for various stimuli. In vivo impulse caused by receptor for stimulation, afferent nerve endings, reach the reactor reaction. Response is excited or inhibited. The relative dose depends on the application of laser. Part of the proper dose of laser energy in certain organisms, as appropriate amount of stimulation answer in response to this, at the molecular level is to adjust the synthesis of protein and nucleic acid, DNA replication, regulation of enzyme function at the cellular level is the mobilization of compensation, nutrition, repair, and other immune defense mechanisms to eliminate the pathological process. The mechanism of laser bio stimulation is a reasoning hypothesis. In foreign countries, the weak laser bio-stimulation mechanism theory is inconsistent. A biological electric field hypothesis, light regulation system hypothesis, cell membrane receptor hypothesis and the hypothesis of the four polarization stimulation is not consistent with the hypothesis. This shows that the theory is not mature and need to be further discussed.

The second mechanism is thermal effect. The thermal effect is the main factor of biological effect.It plays a role in all of the laser irradiation. High power semiconductor laser is mainly thermal effects of its application. Laser in biological tissue, tissue molecular absorption photon energy, the vibration and rotation increased, in the macro performance of the local irradiation becomes hot, high temperature. Because there exist such as melanin, hemoglobin, carotenoids and other pigments in the tissue cells, can increase the light absorption, so that the heat effect of laser is more significant. The interaction between laser and biological is a complicated process determined by various factors. The laser parameters such as wavelength, power, energy, coherence, polarization mode, laser modes have different effects on the biological tissue. The biological tissue properties such as density, elasticity, thermal conductivity, heat capacity, thermal diffusivity, reflectivity, absorption rate, pigment, moisture content, uniformity and hierarchical structure, also will make a different reflection of laser. In recent years, research on laser and biological interaction gradually, mainly discusses the absorption and scattering of laser tissue from theory and experiment, determine the temperature distribution and organization within the organization within the laser energy. From the macroscopic view, it is the study of human optical and laser. Its purpose is to achieve precise control and quantification of laser medical technology and to improve the clinical effect. 


\section{Clinical Applications of Semiconductor Laser}

\section{Applications in Ophthalmology Department}

The eyesare the organswhich can readout the light in the measurement, diagnosis or treatment. Nowadays, laser can play an important role. It is current in the department of ophthalmology in the use and development of about 20 kinds of lasers, which is mainly used for semiconductor laser photocoagulation and treatment of fundus diseases. Low power $810 \mathrm{~nm}$ near infrared semiconductor laser, because the wavelength of the laser penetration, refractive interstitial to absorb at least, and the adjustable range of spot diameter is larger, the most commonly used heat source in the Department of Ophthalmology, can be used for the treatment of various refractory glaucoma after silicone oil injection, refractory and high intraocular pressure, retinal photocoagulation and fixed. Since then, many scholars are exploring aspects of the experimental animal and human eye, studies of semiconductor laser with low energy can penetrate the sclera, colliery body tissue is absorbed, and photocoagulation in epithelium and stromal, the non-pigmented colliery epithelium necrosis, and the real water production function with inhibition or loss, to reduce intraocular pressure. The energy required for the low penetration of sclera, energy can better be melanin absorption, no thermal damage caused by refractive absorbing excessive energy, power and the needed time is low, short. The study found that, the micro pulse semiconductor laser used in ophthalmic therapy relative does not damage the sensory layer of the retina, thereby reducing the occurrence, and better for the opacity of refractive interstitial penetration. But because most of the energy is a semiconductor laser choroid absorption, so laser coagulation reaction is not easy to grasp, easy to cause excessive choroid damage. One time of a photocoagulation treatment cannot achieve satisfactory curative effect and usually requires repeated treatments.

\section{Applications in Surgery Department}

At present, $808 \mathrm{~nm}$ semiconductor laserhas been widely used in low power fusion research organization in the surgical operation. Wolf DeJonge found that, in the semiconductor laser welding vessel welding point average rupture pressure and other commonly used laser center, but the postoperative aneurysm formation of the lowest probability. With the development of laser welding solder, semiconductor laser tissue welding firm is also growing, used in tissue fusion will become more and more widely. Germany's company, especially for PLDD (Percutaneous Laser Disc Decompression) the latest models of minimally invasive operation and production of CeralasD15, which is a type 980nm semiconductor laser knife, in recent years has been from Germany, France and other countries USA physicians as an innovation in the field of use, its clinical application is becoming more and more mature, the treatment effect to get instant results. Beijing Long Huiheng medical science and technology development limited company introduced German technology, pioneered the HOP-100 semiconductor laser operation knife system in the country to fill the gaps in the domestic field of laser medical research blank, the wavelength $830 \mathrm{~nm}$, output power of $0 \sim 30 \mathrm{~W}$, continuously adjustable or intermittent pulse, the output of fiber, air cooling, the size of instrument only $485 \mathrm{~mm} \times 380 \mathrm{~mm} \times 230 \mathrm{~mm}$. It can be widely used in vascular surgery, Department of thoracic surgery, Department of neurosurgery operation in. The present research has shown that $980 \mathrm{~nm}$ semiconductor laser cutting, solidification and other aspects of the application effect is better than the $830 \mathrm{~nm}$ semiconductor laser, and the required power is low. In addition, semiconductor laser can also be used for the treatment of skin herpes zoster, diabetic skin ulcer, and old injury of general surgery diseases.

\section{Applications in Cosmetology Department}

In the field of cosmetic medicine, one of the main applications of laser is the laser hair removal. However, the developed countries in Europe and America have developed quite mature. According to statistics, the global operation of laser hair removal in the range of up to 3.1 million passengers in 2006, an increase of $22 \%$ compared to 2005 and is expected to rise to an annual rate of $18 \%$. $810 \mathrm{~nm}$ semiconductor laser can well be hair follicle melanin absorption, thermal effect, damage the hair follicle, is the gold standard of laser hair removal. At present, the international laser hair removal instrument mainly includes Light sheer, America hair removal Instrument Company in Germany Asclepiusmediate Laser Technology using Light Sheer for laser hair removal operation 800 times in 
250 sites of 144 patients with grade II-V, 84\% parts in 2 after treatment, the effect is very obvious, for the comfort of more than $96 \%$. Acne is a kind of the most common skin diseases in adolescents, the incidence rate of more than $80 \%$. Smooth Beam laser treatment instrument developed by Candela using selective $1450 \mathrm{~nm}$ semiconductor laser to improve the structure of the sebaceous glands under the skin, equipped with power cooling device is very effective to protect the epidermis, face and back acne and acne scar healing treatment. In addition, Anderson1915nm semiconductor laser can selectively be sebaceous gland absorption, heat damage increased sebaceous glands, may be used to cure acne. In recent years, some experts found that the semiconductor laser wrinkle, the laser can penetrate into the dermis layer of the skin collagen cells under the epidermis of about $4 \mathrm{~mm}$, the dermal collagen tissue represents the skin structure become the target tissue for laser irradiation, effectively improve the collagen tissue, the ordered arrangement, regeneration, proliferation and thickening, thereby eliminating wrinkles make the skin becomes smooth and delicate, elastic recovery, and can reduce the complications.Another important application of semiconductor laser in the field of beauty is skin reconstruction operation, for wrinkles, skin rejuvenation. Candela company developed -Smooth Beam semiconductor laser therapeutic instrument, laser wavelength $1450 \mathrm{~nm}$, pulse width is $210 \mathrm{~ms}$, the energy density of $8 J / \mathrm{cm}^{2}$ to $25 \mathrm{~J} / \mathrm{cm}^{2}$, the laser is dermal collagen tissue in water absorption, thermal effect, stimulate regeneration and remodeling of collagen, make skin smooth and delicate, elastic recovery. It can protect the skin tissue with dynamic cooling treatment and reduce the complications.

\section{Applications in Stomatology Department}

Many domestic and foreign literatures show that low intensity laser irradiation has a good curative effect for the treatment of oral ulcer erosive lichen, joint dysfunction and other common chronic oral diseases. Matthias Kreisler et al 809nmAIAS semiconductor laser power output of $10 \mathrm{~mW}$ laser irradiation of human gingival fibroblasts, the exposure time is $75 \sim 300$ s, every $24 \mathrm{~h}$ respectively after 2 times or 3 times. The experiment found that the irradiated cells exhibited a high proliferative activity, the treatment of oral ulcer in the oral cavity and the wound healing is very beneficial in clinical practice, however, continue to observe the proliferation of phenomenon of limited duration, after $24 \mathrm{~h}$ irradiation the cell proliferation activity is very strong, and the proliferation activity gradually decreased in $48 \mathrm{~h}$ and $72 \mathrm{~h}$. This shows that in order to realize the positive role of laser in clinical, repeated treatment is necessary. Stomatitis is false the wearer often suffer from disease. Through clinical research, low intensity laser irradiation can kill harmful bacteria in the mouth, and the bactericidal effect on the gums than on the mucosa obviously. It has important significance for treatment of stomatitis. Many experts and scholars study found, wavelength of inhibitory effects of low intensity laser irradiation on $630 \mathrm{~nm}$ bacteria is the most effective. Temporo Mandibular Disorder is due to masticatory muscle imbalance, abnormal function, structure between the various components of the joint, and even the emergence of organic destruction and produces a series of syndrome, manifested as local chronic pain, accompanied by fatigue, sleep disorders and depression symptoms. The pain caused by medium TMD can lead to inflammation, inflammation and increase the pain itself. The semiconductor laser directly on the deep tissue of the human body and the effective acupuncture point, the local point light stimuli can produce anti-inflammatory, antispasmodic, analgesic, improve microcirculation, regulate immune function and role, so that the correct joint disorder. He spread way of traditional musicis the way as the center is unidirectional. It is spread from the point to the surface. The new media is from point to point, point and surface, and the spread of multi surface and surface interaction. The interactive relationship between the media and the audience, the audience and the audience has established. When the music information communicators will be uploaded to the Internet, the receiver can immediately respond, and timely feedback to the original communicator, can also be forwarded to other people, two-way interaction, interactive information. Therefore, people in the network communication and the audience, also plays a dual role. The music information updated to state communication, real-time bidirectional communication, and timely communication range, more than any previous media. The spread of information including text, sound, images, images, and data symbols can upload, download, copy, print and you can always find it. 


\section{Prospects of Applications of Semiconductor Laser in Medical Fields}

With the development of semiconductor laser technology, laser wavelength range continues to expand. At present, the development of semiconductor laser medical equipment mainly concentrated in developed countries, such as Japan, Europe American, instrument development more miniature, integrated, multi-functional, intelligent direction. China's semiconductor laser treatment is still in the initial stage, the overall level has a big gap compared with the developed countries, the domestic medical equipment mainly rely on imports. However, China has become the second America, Japan is the world's third largest medical laser market, has great potential for development. With the continuous development of semiconductor laser technology in China and the tireless efforts of various disciplines researchers, China's advanced semiconductor laser medical equipment development level will have improved. The overall level of China's medical laser will also be enhanced in the foreseeable future.

\section{References}

[1] Li Yanhua, Kang Zhilong, Hu Liming, New Application of Semiconductor Laser in Medical Field, J. Laser Journal. 6(2010) 73 - 75.

[2] Su Hua, Li Shouchun, Wang Lijun, Applications and Prospect of Semiconductor Laser in Medical Fields, J. Applied Laser. 2(2006) 125 - 129.

[3] Su Hua, Li Shouchun, Applications and Prospect of Semiconductor Laser in Medical Fields, J.Journal of Jilin University (Information Science Edition). 5(2006) 501 - 506.

[4] Yang Xiaoyan,Yang Jiqing, Review of Semiconductor Laser in Medical Applications, J. Chinese Medical Equipment.6(2005)22-23. 